\title{
Wayfinding: The effects of large displays and 3-D perception
}

\author{
Louisa Dahmani • Andrée-Anne Ledoux • \\ Patrice Boyer • Véronique D. Bohbot
}

Published online: 2 November 2011

(C) Psychonomic Society, Inc. 2011

\begin{abstract}
Large displays and stereopsis have been shown to improve performance in several virtual navigation tasks. In the present research, we sought to determine whether wayfinding could benefit from these factors. Participants were tested in a virtual town. There were three viewing conditions: a desktop, a large screen, and a large screen on which the virtual environment was viewed in three dimensions (3-D) using polarized glasses. Participants explored the town and had to remember the location of several landmarks. Their memory of the layout of the town was tested by asking them to navigate from one landmark to another, taking the shortest route possible. All groups performed equally well in terms of the distance traveled to target locations. From this result, we concluded that large displays and 3-D perception do not significantly contribute to wayfinding. Thus, experimental paradigms and training programs that utilize wayfinding are as valuable when administered on standard desktops as on more sophisticated
\end{abstract}

\section{Dahmani $\cdot$ V. D. Bohbot $(\bowtie)$}

Department of Psychiatry, Douglas Mental Health University Institute, FBC Building, McGill University, 6875 boul. LaSalle,

Verdun, Quebec, Canada H4H 1R3

e-mail: veronique.bohbot@mcgill.ca

URL: http://www.bic.mni.mcgill.ca/ vero/

\section{A.-A. Ledoux}

University of Ottawa School of Psychology,

136 Jean-Jacques Lussier,

Ottawa, Ontario, Canada K1N 6N5

\section{P. Boyer}

European Initiative for Research Networks,

Paris University,

1 Place Jussieu,

Paris, France 75005 and costly equipment and do not induce simulator sickness as large displays tend to do.

Keywords Wayfinding · Spatial memory - Virtual navigation $\cdot 3$-D $\cdot$ Large displays $\cdot$ Large screens

The use of virtual environments (VEs) as a research tool has many advantages. Fully controlled settings can be created in which physical aspects such as lights, objects, layouts, and colors can be manipulated (Gamberini, 2000). VEs are especially useful in the study of spatial cognition since they ensure that all participants experience various environments under the same physical conditions. Furthermore, VEs enable navigation through a variety settings within the confines of a single room (Fortenbaugh, Hicks, Hao, \& Turano, 2007).

Large displays are becoming more common with technology advances, and as such, their usefulness has been the subject of several studies. In general, large screens have been used in collaborative tasks, but little has been done to study the benefits of large displays on performance on an individual scale (Tan, Gergle, Scupelli, \& Pausch, 2006), especially in the area of spatial memory.

Using VEs, past studies have found large displays to enhance cognitive map formation (Patrick et al., 2000), object localization (Czerwinski, Tan, \& Robertson, 2002), and wayfinding ability, even when the retinal image size cast by a large screen and a smaller desktop monitor are the same (Tan et al., 2006).

Another factor that may increase performance on VE navigation tasks is 3-D vision, or stereopsis. In two studies, researchers have found that stereoscopic displays, as compared with nonstereoscopic displays, yield greater feelings of presence (Barfield, Hendrix, \& Bystrom, 1999; Lin, 2004), defined as a state of consciousness in which one 
feels as if he or she were present in the virtual environment (Slater \& Usoh, 1993). These increased feelings of presence may enhance wayfinding performance. To our knowledge, the impact of stereopsis on spatial memory has never been directly assessed.

In the present research, we compared three different viewing conditions in order to investigate whether display size and stereopsis have an impact on spatial memory in a virtual wayfinding task. In the first condition, the VE was viewed in two dimensions (2-D) on a standard desktop monitor ("desktop" condition). In the second condition, the VE was viewed in 2-D on a large screen ("large 2-D" condition), and in the third condition, the VE was viewed in 3-D on a large screen ("large 3-D" condition) using filtered glasses. We chose not to keep the visual angle constant across conditions in order to study the effect of having the same visual information cover different amounts of the visual field. We hypothesized that experiencing the VE on a large screen would lead to better wayfinding performance, and as such, we expected the group viewing the VE on a large screen to show better results than the one viewing the VE on a standard desktop monitor. We also predicted that navigation performance would be enhanced in the 3-D large-screen condition as compared with the other conditions because of the added effects of stereopsis.

\section{Method}

\section{Participants}

Seventy-four healthy university students and hospital staff (38 women, 36 men) were tested. All participants were righthanded and had no history of neurological or psychiatric disorders. Testing took place in the Memory \& Motion Laboratory at the Douglas Mental Health University Institute. Informed consent was obtained in conformity with the local ethics committee requirements. Participants received monetary compensation or course credit for their participation. Eight participants withdrew from the study because of simulator sickness. Of these, five withdrew while in the exploration phase, and three were able to complete some of the test trials ("probe" trials) that are used to assess wayfinding performance. The data for the individuals who completed some of the probe trials were kept for the analyses. In addition, one participant was excluded because the criteria for exploration of the town could not be reached during the exploration phase, one participant was excluded because their scores exceeded three standard deviations above the mean on all measures, and one participant was left-handed. Thus, 66 people were included in the final analyses (34 women, 32 men). Participants were assigned to one of three viewing conditions. In one condition, participants performed the task on a standard desktop computer and viewed the virtual environment in 2-D (desktop condition). In a second condition, the VE was viewed in 2-D on a large screen (large 2-D condition). In a third condition, the VE was viewed in 3-D on a large screen (large 3-D condition). Twenty-five participants were included in the desktop condition (13 women, 12 men), 21 in the large 2-D condition, (11 women, 10 men), and 20 in the large 3-D condition (10 women, 10 men).

\section{Apparatus}

The large screen conditions used a rear-projection screen apparatus, consisting of an Epson projector and a $3 \mathrm{~m}$ wide $\times 2.30 \mathrm{~m}$ tall screen. The screen resolution was $1,600 \times 1,200$. The desktop monitor used in the desktop condition was a standard 17-in. Viewsonic monitor, with a 1,280 × 1,024 resolution. Both screens provided clear images with no blurring. In the large 3-D condition, a dual projection system with polarizing filters and stereoscopic glasses was used to view the environment in three dimensions. The horizontal visual angle subtended by the desktop screen was approximately $27^{\circ}$, whereas that of the large screen was approximately $62^{\circ}$.

The practice and virtual town maps were created using the Unreal Tournament 2003 (Unreal Tournament 2003; Epic Games, Raleigh, NC) computer game development platform. Two previously described virtual maps (Etchamendy \& Bohbot, 2007) were used for this study: one practice map and one experimental virtual town map (Figs. 1 and 2). The virtual town contained eight key landmarks such as shops, a school, a hospital, and so on.

In order to measure participants' IQ, the Shipley Institute of Living Scale (WPS, 2000) was administered. The test consists of a vocabulary section and an abstraction section. A time limit of $10 \mathrm{~min}$ was given for each section. Scores obtained on this test can range from 0 to 80 points. An estimated WAIS-R IQ for all but five participants was determined from the Shipley by using a conversion table based on the score and age of the participant.

\section{Procedure}

The administration of the virtual task on the large screen was found to cause dizziness and nausea in approximately a third of participants in previous studies (Bohbot, personal communication); therefore, participants were asked in a prescreening questionnaire whether they felt ill in a variety of situations. If they were prone to feelings of nausea, they were excluded from the large display conditions (large 2-D and large 3-D), since these are more immersive and more likely to cause nausea, and were assigned to the desktop condition. If they were not prone to feelings of sickness, 
Fig. 1 Two screenshots of the virtual town. The first one represents a shop, and the second one shows an aerial view of the map (screenshots borrowed from Etchamendy \& Bohbot, 2007)

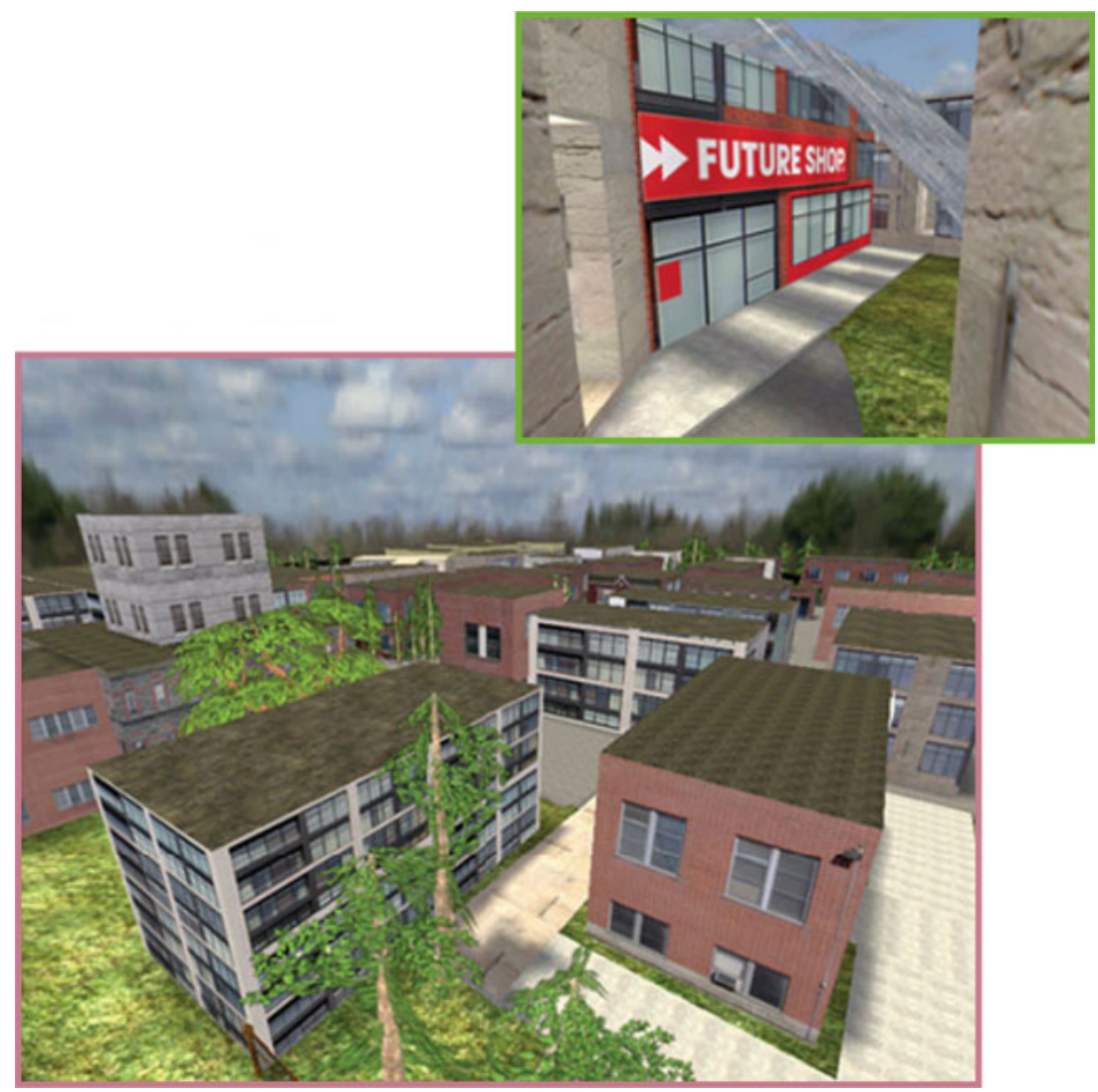

then they were randomly assigned to one of the two large display conditions. This was done in an attempt to minimize the number of participants who would abort the

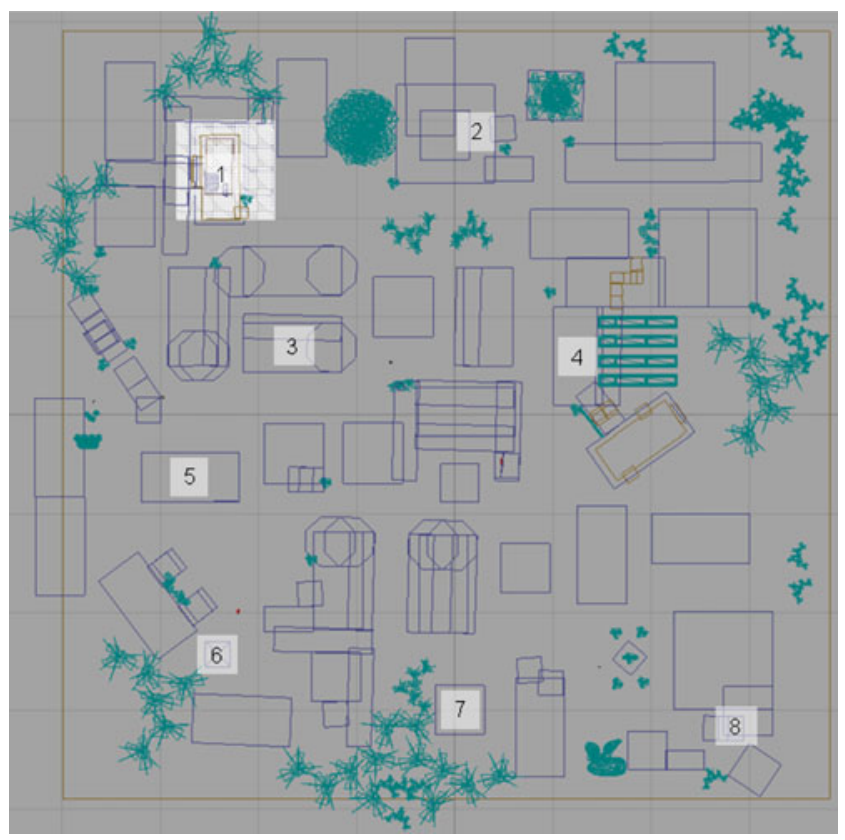

Fig. 2 A bird's eye view of the virtual town. The town comprises various buildings, streets, alleys, and eight key landmarks, indicated with numbers experiment because of simulator sickness. Next, they were placed in a practice virtual environment to familiarize themselves with the keys that allowed them to move around. They were instructed to navigate in the virtual environment using the forward, left, and right arrow keys on a keyboard. In order to best simulate real-world navigation, participants were not allowed to navigate using the backward key. Participants were seated during the VE tasks. Additionally, in the large 3-D condition, participants wore a pair of polarized glasses in order to view the fully colored maps in three dimensions. When both the experimenter and participant agreed that the participant was comfortable enough navigating with the keys, the experimental task was given.

Participants freely explored the virtual town for a minimum of $20 \mathrm{~min}$. The virtual town was approximately $151 \times 153$ in virtual meters $\left(23,103 \mathrm{~m}^{2}\right)$. It included various streets, alleys, buildings, and eight important landmarks that were clearly identified (e.g., pool, school, hospital; Figs. 1 and 2). The key landmarks were placed in the town so that, from a given landmark, no other landmark would be visible. Doing so prevented the formation of stimulusresponse associations between the different landmarks in the short exploration period that was allowed; thus, participants could not use an egocentric strategy to learn their way between different landmarks. Participants were 
instructed to go down every street in the town and to learn the location of eight specific landmarks. They were told that they had to remember the location of these landmarks because they would be asked to navigate to some of them later on. The landmarks were brought to their attention the first two times they were encountered. Intervention by the experimenter was sometimes needed to ensure that participants visited each landmark at least twice and that they took all the pathways at least once by the end of the exploration phase. The exploration phase was ended after these criteria were reached, and the exploration lasted at least $20 \mathrm{~min}$. This exploration phase allowed participants to construct a cognitive map, formed by building relationships between landmarks in the environment (O'Keefe \& Nadel, 1978; Tolman, 1948).

Eight probe trials were then administered to assess the degree to which participants were able to form a cognitive representation of the town. For each of the eight probe trials, participants were placed in front of one of the landmarks and were instructed to navigate to another landmark by taking the most direct route possible, and to not worry about the time they took to complete the trial. They nonetheless had a time limit of $5 \mathrm{~min}$ to complete each trial. The start and finish landmarks were never repeated, to ensure that the paths taken were relatively unfamiliar for each of the probe trials. The route taken was drawn by the experimenter on a 2-D aerial view of the town printed on a sheet of paper, and the time to reach the target location was recorded.

\section{Analysis}

For the analyses, we considered only successful trials. These were defined as trials that were completed within three times the shortest time it takes to reach the target location on a given trial. This was done to minimize the impact of unsuccessful trials (in which the target location was not found within the 5-min time limit) or trials on which the target location was found by chance on the means of the various wayfinding variables. Unsuccessful trials were taken into account by the percentage of target locations found variable. The dependent variables thus included mean distance traveled (measured by the length in centimeters of the route drawn on the 2-D aerial view of the town), mean time, and mean percentage of target locations found.

\section{Results}

The average shortest route between two landmarks was $10.76 \mathrm{~cm}$. In comparison, the mean distance participants traveled in the probe trials was $14.5 \mathrm{~cm}$. Each group's mean distance traveled, mean distance error, mean time, and mean percentage of target locations found are shown in Table 1, along with participant demographics. In order to verify whether the three groups (desktop, large 2-D, and large 3-D) performed differently in the wayfinding task inside the experimental virtual town map, a MANOVA was performed, with mean distance traveled, mean distance error, and mean time as the dependent variables, and with viewing condition as the between-subjects factor. No main effect of condition was found, $F(4,124)=0.33, p>.05$. The three groups therefore did not differ in terms of mean distance traveled, $F(2,63)=0.13, p>.05$ (Fig. 3a), or mean time, $F(2,63)=0.53, p>.05$ (Fig. 3b). A one-way ANOVA revealed no significant difference between groups in the mean percentage of targets found, $F(2,63)=1.68, p>.05$ (Fig. 3c).

Multiple regression analyses were performed to determine whether wayfinding performance could be predicted from age, sex, IQ, number of years of education, or

Table 1 Participant characteristics and performance measures in each condition. Means are shown with standard deviations in brackets. The groups did not differ in terms of characteristics or performance

\begin{tabular}{|c|c|c|c|c|c|}
\hline & Desktop & Large 2-D & Large 3-D & $F$ & $\eta^{2}$ \\
\hline \multicolumn{6}{|l|}{ Participant characteristics } \\
\hline$N$ & 25 & 21 & 20 & & \\
\hline Women:Men & $13: 12$ & $11: 10$ & $10: 10$ & 0.01 & \\
\hline Age (years) & $23.8[4.8]$ & $22.5[3.9]$ & $22.1[2.5]$ & 1.14 & \\
\hline Number of years of education & $16.2[2.7]$ & $16.3[2.1]$ & $15.9[5.5]$ & 0.26 & \\
\hline WAIS-R IQ & $111.9[6.2]$ & $108.8[5.5]$ & $107.3[11.1]$ & 1.74 & \\
\hline \multicolumn{6}{|l|}{ Wayfinding Task } \\
\hline Mean exploration time (in seconds) & $1232[66.1]$ & $1228[68.1]$ & $1222[54.4]$ & 0.12 & \\
\hline Mean distance (in centimeters) & $14.96[2.2]$ & $14.59[2.4]$ & $14.78[2.6]$ & 0.13 & 0.004 \\
\hline Mean time (in seconds) & $64.74[15.1]$ & $59.85[13.7]$ & $62.57[19.1]$ & 0.53 & 0.017 \\
\hline Mean percentage of targets found (in percentages) & $83.5[21.3]$ & $93.5[13.5]$ & 86.9 [19.2] & 1.68 & 0.051 \\
\hline
\end{tabular}


Fig. 3 Means for the desktop, large 2-D, and large 3-D groups are shown in terms of a distance traveled (in centimeters), $\mathbf{b}$ time (in seconds), and c percentage of target locations found. The three groups do not significantly differ in any of these measures, $p>.05$. The error bars represent standard errors of the mean
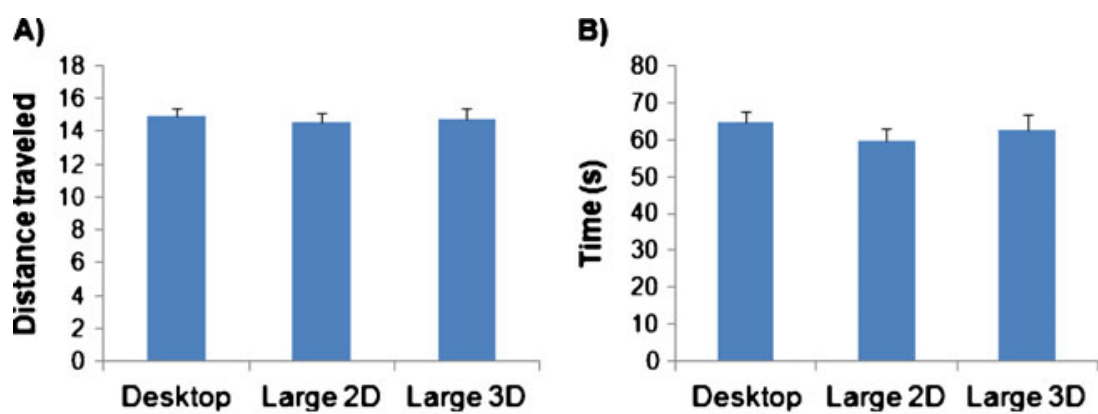

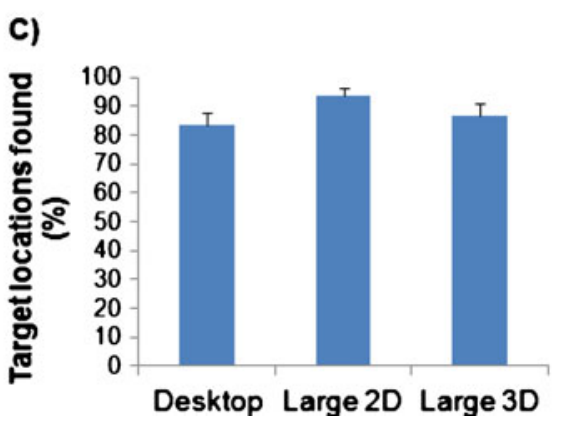

exploration time. Mean distance traveled and percentage of targets found were taken as the dependent variables. This combination of variables did not significantly predict the mean distance traveled, $F(5,55)=1.31, p>.05$. However, these variables significantly predicted the mean percentage of targets found, $F(5,55)=4.66, p<.005$. Education, $t=$ 2.76, $p<.01$, and exploration time, $t=-3.15, p<.01$, significantly contributed to the prediction, whereas there was a tendency toward significance for age to also contribute, $t=-1.79, p=.078$. The adjusted $R^{2}$ was .23 , indicating that $23 \%$ of the variance in percentage of targets found is explained by the model, which represents a medium to large effect size according to Cohen (1988). The beta weights suggest that number of years of education contribute most to predicting percentage of targets found $(\beta=$ $0.43)$, and that being younger $(\beta=-0.29)$ and exploring the virtual town for a less amount of time $(\beta=-0.39)$ also contribute to a higher number of targets found. In order to gain a better understanding of the fact that exploration time negatively correlated with percentage of targets found, we correlated the times participants passed by a landmark during the exploration phase with exploration time. The data available for 23 participants showed a negative correlation that almost reached significance, $r=-0.38, p=.07$. Thus, there is a tendency for people who take longer to reach criteria in the exploration phase to visit landmarks fewer times - a finding that could help researchers to understand why participants with a longer exploration time have a lower percentage of targets found.

Four one-way ANOVAs were conducted to examine whether participants in the three viewing conditions differed in sex, age and education, IQ, or exploration time.
No significant differences were found between groups in terms of these variables, $p>.05$.

Finally, upon observing that many participants in the large-screen conditions felt simulator sickness (20/47 compared to $1 / 27$ in the desktop condition), we compared participants with and without nausea to determine whether it had an effect on wayfinding performance using a MANOVA. No significant difference in performance was found, $p>.05$. We also ran a separate analysis that considered only people who did not experience simulator sickness. We did not find significant differences between groups in any of the wayfinding variables, $p>.05$.

Finally, we also looked at whether sex modulated wayfinding performance by conducting a MANOVA. A marginally significant main effect of sex was found, $F(2,63)=2.91$, $p=.062, \eta^{2}=0.085$ (medium effect size). The univariate ANOVAs reveal that men significantly traveled shorter distances, $F(1,64)=5.74, p<.05, \eta^{2}=0.082$ (medium effect size), and were faster at finding targets, $F(1,64)=4.60$, $p<0.05, \eta^{2}=0.067$ (medium effect size), than women. An independent samples $t$ test was performed for mean percentage of targets found; no significant difference between men and women was found, $t(65)=-0.91, p>.05$.

\section{Discussion}

We did not find a significant difference between viewing conditions in distance traveled, time to reach target location, or percentage of target locations found. We conclude that large screens and 3-D perception do not enhance memory in wayfinding tasks. Similarly, other 
studies in the literature did not find an advantage in large display conditions (Gamberini, 2000; Tan et al., 2006; Tan, Gergle, Scupelli, \& Pausch, 2004), some even finding a significant advantage with a standard desktop monitor (Czerwinski et al., 2002; Gamberini, 2000). There is also evidence showing that large displays can be helpful when using other types of virtual navigation tasks (Patrick et al., 2000; Tan et al., 2004; Tyndiuk, Lespinet-Najib, Thomas, $\&$ Schlick, 2007). For example, Tan et al. (2004) found that large displays enhance performance in a path integration task, in which participants are led along two legs of a triangle and are asked to go back to the start position of the triangle. Participants in the large display group were better able to judge the distance to the start position. However, there was no difference in the angle error, indicating that participants in both the small and large displays were equal in judging the direction in which the start position was located. Similarly, Tyndiuk et al. (2007) gave their participants three different tasks: a prime search task, in which they had to move to a visible chair in the environment; a naive search task, in which they had to find invisible chairs; and a manipulation task, in which they had to orient a chair in such a way that it would stand on its legs. Large displays were found to aid performance in the naive search task and the manipulation task. Additionally, route learning was also found to be enhanced with the use of large displays. Patrick et al. (2000) verbally instructed their participants to go from one ride to another in a virtual amusement park along a fixed route. Participants in the large-screen condition were more accurate than participants in the monitor condition when asked to reproduce the map of the amusement park. Thus, performance on several navigation tasks was found to be enhanced with the use of large displays.

The impact of large displays on virtual navigation is mixed, however, since there are studies that did not find larger displays to be beneficial. For example, Gamberini (2000) investigated object location using a desktop monitor and a head-mounted display, which offers a wider field of view. Participants had to explore a virtual lounge and pay attention to objects within it. They were then given object location and recognition probes. There was no difference between the desktop and the head-mounted display with regard to object location. Surprisingly, object recognition was found to be better in the desktop condition. Furthermore, Tan et al. (2006) investigated mental rotation and found no effect of display size. Thus, large displays are not always beneficial.

Very few studies have looked at wayfinding and display size. Bakdash, Augustyn and Proffitt (2006) conducted a study in which participants explored a virtual environment and had to learn the location of five objects on either a small or a large display. In the test phase, participants wore head-mounted displays and were placed at each of the five target objects. The task was to point in the direction of each of the other four targets. Participants in the large display group exhibited smaller angular pointing errors than did the participants in the small display group. A study by Czerwinski et al. (2002) in which participants had to learn and point to the direction of four objects in a virtual environment yielded similar results. However, they found that the large-screen condition actually induced participants to travel longer distances than in the small display condition. On the other hand, Tan et al. (2006) administered a wayfinding task in which participants explored two virtual environment and were asked to find the shortest distance to a given target from a specific point. They found that participants tested on a large screen traveled shorter distances. Discrepant results may be the product of a number of methodological differences between studies such as virtual environment size, number of landmarks, exploration period, and complexity. All of these factors play a role because they influence cognitive map formation, and make it difficult to determine in which situations large displays enhance performance. Therefore, it would seem that there are conflicting results regarding wayfinding enhancement and large displays.

It is also important to note that many of these studies had a constant field of view across viewing conditions - that is, the larger the display, the further it was placed from the participant in order for the viewing angle to be the same as in the small display condition. In our study, the large screen covered most of the field of view, which concurrently induced simulator sickness (Lestienne, Soechting, \& Berthoz, 1977; Seay, Krum, Hodges, \& Ribarsky, 2002). Since simulator sickness may have had a negative impact on wayfinding performance, an analysis that included only participants who did not experience simulator sickness was carried out. No significant differences between the three conditions were found. Comparing participants with and without nausea did not reveal significant differences in performance either, indicating that simulator sickness had no impact on performance.

Tyndiuk et al. (2007) argued that not everyone benefits from cognitive aids such as large displays. Indeed, they found that only participants with low levels of attentional ability showed enhanced performance on large versus desktop displays on their prime and naive search tasks. Participants with high levels of attentional ability performed equally well on the two display types. Tyndiuk et al. tested high school students, which may have brought more variety in attentional abilities. Since our participant pool consisted mainly of university students, we suggest that they had higher attentional abilities a priori, and that this translated into equal performance in the desktop and large displays. 
There was also no significant effect of stereopsis on wayfinding ability. This finding was in accordance with that of Hale and Stanney (2006), who did not find improved navigation efficiency as a result of greater stereoscopic acuity. The authors tested participants with either low or high stereoscopic acuity while wearing a head-mounted display on a variety of manipulation tasks, in which they had to grasp and drag various objects to specific places, and on locomotion tasks, in which they had to move through different rooms and hallways. A difference was found between groups, in which low stereoscopic acuity resulted in poorer navigation efficiency. However, the improvement was minimal and was found only in two of the seven tasks. The authors concluded that overall performance was comparable between the low and high stereo acuity groups, a finding that supports our results.

Besides stereopsis, object interposition, size constancy, linear perspective, relative size, and motion parallax are all pictorial and movement-based cues that aid depth perception (Hale \& Stanney, 2006). Since all of these depth cues are present in the conditions in which the perception is nonstereoscopic, we suggest that the depth information received in the nonstereoscopic conditions (desktop and large 2-D) was sufficient to efficiently perform the wayfinding task. This result is supported by a previous study showing that spatial perception did not benefit from stereopsis when visual scenes already provided motion parallax (Davis \& Hodges, 1995). Hale and Stanney also measured feelings of presence and found no difference between the two groups. The authors suggested that motion parallax may have a greater impact on sense of presence than stereopsis (Hale \& Stanney, 2006). Hence, stereopsis does not seem to appreciably enhance performance on virtual navigation tasks and appears to be unnecessary, since many other depth cues are provided within 3-D environments.

Several variables were shown to be linked to wayfinding performance. Education was found to be positively associated with the percentage of targets found, whereas exploration time and age were negatively associated with this variable. It may seem peculiar that exploration time negatively correlated with the percentage of targets found in the probe trials. However, since the exploration phase was ended when participants reached the criteria of visiting each of the eight landmarks twice and taking every road at least once, it is possible that people who have more difficulty orienting in space and learning the relationships between landmarks are slower and need more time to explore every pathway in a town. This result is supported by the finding that the longer the exploration time, the fewer times landmarks were visited. Therefore, many factors, such as education, have an impact on wayfinding performance and should be taken into account when studying wayfinding.
When exploring sex differences, we found that men outperformed women in the wayfinding task: They found more direct routes to target locations and took less time. However, men and women did not differ in the number of landmarks that they found, showing that women are slower and find longer shortcuts than men, but that they are able to find target locations equally as often. Few studies have looked at free exploration wayfinding and sex. Most studies lead participants along a route and ask them to reproduce this route (Postma, Jager, Kessels, Koppeschaar, \& van Honk, 2004) or to find the shortest route between landmarks or locations along this route (Kober \& Neuper, 2011; Silverman et al., 2000), which yields conflicting results in which sex differences sometimes emerge and sometimes do not. One study (Sakthivel, Patterson, \& Cruz-Neira, 1999) required students to navigate from one place to another within a virtual version of their school campus. Various measures were recorded such as distance traveled, time, number of wrong turns, and so on, and men were found to consistently outperform women. Another study (Astur, Tropp, Sava, Constable, \& Markus, 2004) used the Morris Water Maze paradigm in which participants learn the location of a hidden platform that will take them out of a pool. Visual cues outside of the pool can help find the location of the hidden platform. As in our study, men took less time than women to find the hidden platform and traveled shorter distances. Thus, it would seem that men are better able to find shortcuts to specific locations and are faster than women. However, we found in our study that women find just as many target landmarks. Similarly, Astur et al. (2004) found that women were as accurate as men at finding target locations when tested in a virtual radial maze, albeit slower. Consequently, the sex differences observed in speed and path length in Astur's study and in our study do not seem to be related to knowledge of target locations but instead must be related to other factors such as differences in navigational preferences (Dabbs, Chang, Strong, \& Milun, 1998; Lambrey \& Berthoz, 2007; Malinowski, 2001; Postma et al., 2004; Ruggiero, Sergi, \& Iachini, 2008; Sandstrom, Kaufman, \& Huettel, 1998; Saucier et al., 2002).

In summary, a large display size and 3-D perception did not improve wayfinding performance relative to using a standard desktop monitor. Our data suggest that studies and intervention programs that employ wayfinding as a tool may not need to invest large physical and financial resources into the acquisition and installation of sophisticated equipment such as large screens, projection systems, and the implementation of 3-D. Moreover, large displays induce simulator sickness in a large proportion of participants, complicating the study for both the experimenters and participants. Our data suggest that learning the layouts of virtual environments can successfully be done through the use of standard desktops. 
Author Note The present research was sponsored by NSERC, Grant 239896. We wish to thank the Canadian Foundation for Innovation for an infrastructure grant that funded the laboratory equipment, CFI Project no: 9357. We wish to thank Kyoko Konishi, Rosalind Sham, and Anna Trowbridge for their help with the manuscript.

\section{References}

Astur, R. S., Tropp, J., Sava, S., Constable, R. T., \& Markus, E. J. (2004). Sex differences and correlations in a virtual Morris water task, a virtual radial arm maze, and mental rotation. Behavioural Brain Research, 151(1-2), 103-115.

Bakdash, J. Z., Augustyn, J. S., \& Proffitt, D. R. (2006). Large displays enhance spatial knowledge of a virtual environment. Paper presented at the Proceedings of the 3rd Symposium on Applied Perception in Graphics and Visualization, Boston, Massachussetts. July

Barfield, W., Hendrix, C., \& Bystrom, K. (1999). Effects of stereopsis and head-tracking on performance using desktop virtual environments displays. Presence, 8, 237-240.

Cohen, J. (1988). Statistical power and analysis for the behavioral sciences (2nd ed.). Hillsdale: Erlbaum.

Czerwinski, M., Tan, D. S., \& Robertson, G. G. (2002). Women take a wider view. In Proceedings of the CHI 2002 Conference on Human Factors in Computing Systems. Paper presented at the CHI 2002 Conference on Human Factors in Computing Systems, Minneapolis, MN (April 2002). (pp. 195-202).

Dabbs, J. M., Chang, E. L., Strong, R. A., \& Milun, R. (1998). Spatial ability, navigation strategy, and geographic knowledge among men and women. Evolution and Human Behavior, 19, 89-98.

Davis, E. T., \& Hodges, L. F. (1995). Human stereopsis, fusion, and stereoscopic virtual environments. In W. Barfield \& T. A. Furness (Eds.), Virtual environments and advanced interface design (pp. 145-174). New York: Oxford University Press.

Etchamendy, N., \& Bohbot, V. D. (2007). Spontaneous navigational strategies and performance in the virtual town. Hippocampus, 17, 595-599.

Fortenbaugh, F. C., Hicks, J. C., Hao, L., \& Turano, K. A. (2007). A technique for simulating visual field lossed in virtual environments to study human navigation. Behavior Research Methods, $39,552-560$.

Gamberini, L. (2000). Virtual reality as a new research tool for the study of human memory. Cyberpsychology \& Behavior, 3, 337-342.

Hale, K. S., \& Stanney, K. M. (2006). Effects of low stereo acuity on performance, presence, and sickness within a virtual environment. Applied Ergonomics, 37, 329-339.

Kober, S. E., \& Neuper, C. (2011). Sex differences in human EEG theta oscillations during spatial navigation in virtual reality. International Journal of Psychophysiology, 79, 347-355.

Lambrey, S., \& Berthoz, A. (2007). Gender differences in the use of external landmarks versus spatial representations updated by selfmotion. Journal of Integrative Neuroscience, 6, 379-401.

Lestienne, F., Soechting, J., \& Berthoz, A. (1977). Postural readjustments induced by linear motion of visual scenes. Experimental Brain Research, 28, 363-384.
Lin, J. J. (2004). Enhancement of user-experiences in immersive virtual environments that employ wide-field displays. Seattle, Washington: University of Washington.

Malinowski, J. C. (2001). Mental rotation and real-world wayfinding. Perceptual and Motor Skills, 92, 19-30.

O'Keefe, J., \& Nadel, L. (1978). The hippocampus as a cognitive map. Oxford, England: Clarendon Press.

Patrick, E., Cosgrove, D., Slavkovic, A., Rode, J. A., Verratti, T., \& Chiselko, G. (2000). Using a large projection screen as an alternative to head-mounted displays for virtual environments. In Proceedings of the CHI 2000 Conference on Human Factors in Computing Systems (pp. 478-485)

Postma, A., Jager, G., Kessels, R. P., Koppeschaar, H. P., \& van Honk, J. (2004). Sex differences for selective forms of spatial memory. Brain and Cognition, 54, 24-34.

Ruggiero, G., Sergi, I., \& Iachini, T. (2008). Gender differences in remembering and inferring spatial distances. Memory, 16, 821835.

Sakthivel, M., Patterson, P. E., \& Cruz-Neira, C. (1999). Gender differences in navigating virtual worlds. Biomedical Sciences Instrumentation, 35, 353-359.

Sandstrom, N. J., Kaufman, J., \& Huettel, S. A. (1998). Males and females use different distal cues in a virtual environment navigation task. Brain Research. Cognitive Brain Research, 6, 351-360.

Saucier, D. M., Green, S. M., Leason, J., MacFadden, A., Bell, S., \& Elias, L. J. (2002). Are sex differences in navigation caused by sexually dimorphic strategies or by differences in the ability to use the strategies? Behavioral Neuroscience, 116, 403-410.

Seay, A. F., Krum, D. M., Hodges, L., \& Ribarsky, W. (2002). Simulator sickness and presence in a high field-of-view virtual environment. Paper presented at the CHI 2002 Extended Abstracts on Human Factors in Computing Systems Minneapolis, Minnesota. April

Silverman, I. I., Choi, J., Mackewn, A., Fisher, M., Moro, J., \& Olshansky, E. (2000). Evolved mechanisms underlying wayfinding. Further studies on the hunter-gatherer theory of spatial sex differences. Evolution and Human Behavior, 21, 201-213.

Slater, M., \& Usoh, M. (1993). Presence in immersive virtual environments. Paper presented at the Proceedings of the IEEE Conference-Virtual Reality Annual International Symposium. September Seattle, WA

Tan, D. S., Gergle, D., Scupelli, P. G., \& Pausch, R. (2004). Physically large displays improve path integration in $3 D$ virtual navigation tasks. Paper presented at the Proceedings of SIGCHI Conference on Human Factors in Computing Systems 2004, Vienna, Austria. April

Tan, D. S., Gergle, D., Scupelli, P., \& Pausch, R. (2006). Physically large displays improve performance on spatial tasks. $A C M$ Transactions on Computer-Human Interaction, 13, 71-99.

Tolman, E. C. (1948). Cognitive maps in rats and men. Psychological Review, 55, 189-208.

Tyndiuk, F., Lespinet-Najib, V., Thomas, G., \& Schlick, C. (2007). Impact of tasks and users' characteristics on virtual reality performance. Cyberpsychology \& Behavior, 10, 444-452. 